\title{
Observações sobre a ocorrência de Mosca-Negra- dos-Citros, Aleurocanthus woglumi Ashby, 1915 (Hemiptera: Sternorrhyncha: Aleyrodidae) no estado do Amazonas
}

\author{
Beatriz RONCHI-TELES ${ }^{1}$, Marcia Reis PENA², Neliton Marques SILVA ${ }^{3}$
}

RESUMO

A mosca-negra-dos-citros (Aleurocanthus woglumi Ashby) é uma importante praga dos citros de origem asiática. Foi detectada no Brasil pela primeira vez em Belém-PA em 2001. Este trabalho tem como objetivo registrar a ocorrência de mosca-negra-doscitros no estado do Amazonas, sua distribuição geográfica e estudos de biologia em condiçóes de laboratório. A mosca-negra encontra-se atualmente disseminada em mais da metade dos municípios paraenses. No Amazonas foi detectada em junho de 2004 em Manaus e atualmente encontra-se disseminada em toda a área urbana deste município, ocorrendo também em Itacoatiara, Rio Preto da Eva e Iranduba. Em observações feitas em condições de laboratório em Manaus-AM, foi verificado que o ciclo de ovo-adulto foi de 71,76 22,07 dias, caracterizando como uma espécie multivoltina.

PALAVRAS-ChaVE: Amazônia, Praga dos citros, Distribuição, Aleirodídeo.

\section{Observation on the occurrence of the citrus blackfly Aleurocanthus woglumi Ashby, 1915 (Hemiptera: Sternorrhyncha: Aleyrodidae) in the Amazonas state}

\section{ABSTRACT}

The citrus blackfly Aleurocanthus woglumi Ashby, pest of citrus in Asian is considered important pest. It was detected for the first time in Belém, PA in 2001. The objective of this work was to report occurrence of the citrus blackfly in Amazon state. Nowadays is found in the majority of the oriental amazon counties. In Manaus, Amazonas was detected in June 2004, actually disseminated in the urban area and in Itacoatiara, Rio Preto da Eva and Iranduba counties. In observations in laboratory in Manaus-AM, was verified that the egg-adult cycle belonged to 71,76 $\pm 2,07$ days, characterizing as a multivoltin species.

KEYWORDS: Amazon region, citrus pest, distribution, Aleyrodidae.

\footnotetext{
1 Instituto Nacional de Pesquisas da Amazônia, INPA. Coordenação de Pesquisas em Entomologia. Av. André Araújo, 2936, Aleixo.CEP 69060-001, Manaus - AM. e-mail: ronchi@inpa.gov.br

2 Universidade Federal do Amazonas, Faculdade de Ciências Agrárias, Laboratório de Entomologia Agrícola, Mini Campus. CEP 69070-000. e-mail: marciarpena@yahoo.com.br

3 Universidade Federal do Amazonas, Faculdade de Ciências Agrárias, Laboratório de Entomologia Agrícola, Mini Campus. CEP 69070-000. e-mail: nmarques@ufam.edu.br
} 
A mosca-negra-dos-citros Aleurocanthus woglumi Ashby é ima importante praga dos citros de origem asiática (Dietz \& Zetek 1920). Encontram-se disseminada nas Américas, África, Ásia e Oceania (Oliveira et al., 2001). Recém introduzida no país, foi detectada pela primeira vez no estado do Pará em 2001 na área urbana do município de Belém. Este trabalho tem como objetivo registrar a ocorrência da mosca-negra-doscitros no estado do Amazonas, sua distribuição geográfica e estudos de biologia em condiçôes de laboratório.

A mosca-negra pode ser encontrada atualmente em mais da metade dos municípios paraenses (Maia et al., 2005). Há também registros de ocorrência nos estados do Maranhão em 2003 (Lemos et al., 2006), Amapá em 2006 (Jordão \& Silva, 2006) e São Paulo em 2008 ((Pena et al., 2008).

Apesar de ser um inseto praga de recente introdução no Brasil (Oliveira et al., 2001), o primeiro registro no novo mundo foi na Jamaica em 1913, tendo se propagado para Cuba em 1916, México em 1935 (Smith et al., 1964). Na Flórida foi registrada em 1934 (Newell \& Brown 1939). Na América do Sul foi detectada em 1965 na Venezuela (Angeles et al., 1968).

No Amazonas foi detectada em Manaus em junho de 2004 sobre plantas cítricas e encontra-se disseminada por toda a área urbana do município de Manaus. Atualmente é encontrada também nos municípios de Itacoatiara, Rio Preto da Eva e Iranduba, registros feitos em visitas nos plantios de citros (Pena \& Silva, 2007).

Trata-se de uma praga de hábito alimentar polífago, sendo citrus seu hospedeiro favorito. São relatadas cerca de 300 plantas hospedeiras deste inseto incluindo-se dentre elas, manga, uva, citros, caju, abacate, goiaba, maçã, figo, banana, mamão, pêra, romã, marmelo, café, rosas, entre outras (Nguyen \& Hamon, 2003). Na região urbana de Manaus, tem sido encontrada infestando folhas de citros (Figura 1A), mangueiras e café.

As observações sobre a biologia foram realizadas no Laboratório de Entomologia Agrícola da Universidade Federal do Amazonas - UFAM, Manaus-AM, no período de março a junho de 2006. Em condiçōes de laboratório $\left(26 \pm 2^{\circ} \mathrm{C}\right.$; UR $80 \pm 3 \%$ e fotofase de 12 horas) foi acompanhado o ciclo de ovo-adulto em três mudas de lima ácida Thaiti, C. latifolia (Yu Tanaka) Tanaka Burm. (porta enxerto Citromelo), cada muda com 60 ovos, estes observados diariamente com auxílio de um microscópio estereoscópico. Foi verificado que os ovos em forma de bastonetes recurvados são colocados em espiral e fixo, por meio de um pedúnculo, na face inferior da folha, são amarelo-claros tornando-se amarelo-escuros quando próximo à eclosão das ninfas (Figura 1B). O período de desenvolvimento embrionário foi de $14,43 \pm 0,75$ dias. Ninfas de primeiro instar duram 9,06 $\pm 0,2$, são móveis, apresentando corpo alongado, com as extremidades enegrecidas; apresentando dois filamentos na região anterior e dois na região posterior. Ninfas de segundo instar são ápodas, assim como as demais e duram $6,76 \pm 0,09$ dias. Apresentam corpo ovalado com presença de cerdas no dorso. Ninfas de terceiro instar duram $8,28 \pm 0,26$ dias; assemelham-se às do segundo, porém as cerdas são mais visíveis e o corpo é enegrecido. Ninfas de quarto instar duram $33,58 \pm 2,12$ dias, são bem ovaladas de corpo negro brilhante. Os adultos apresentam as asas azul-acinzentadas brilhantes; corpo alaranjado com tons de cinza escuro e abdome cinza claro na extremidade. O ciclo de ovo-adulto foi de $71,76 \pm 2,07$ dias, caracterizando como uma espécie multivoltina.
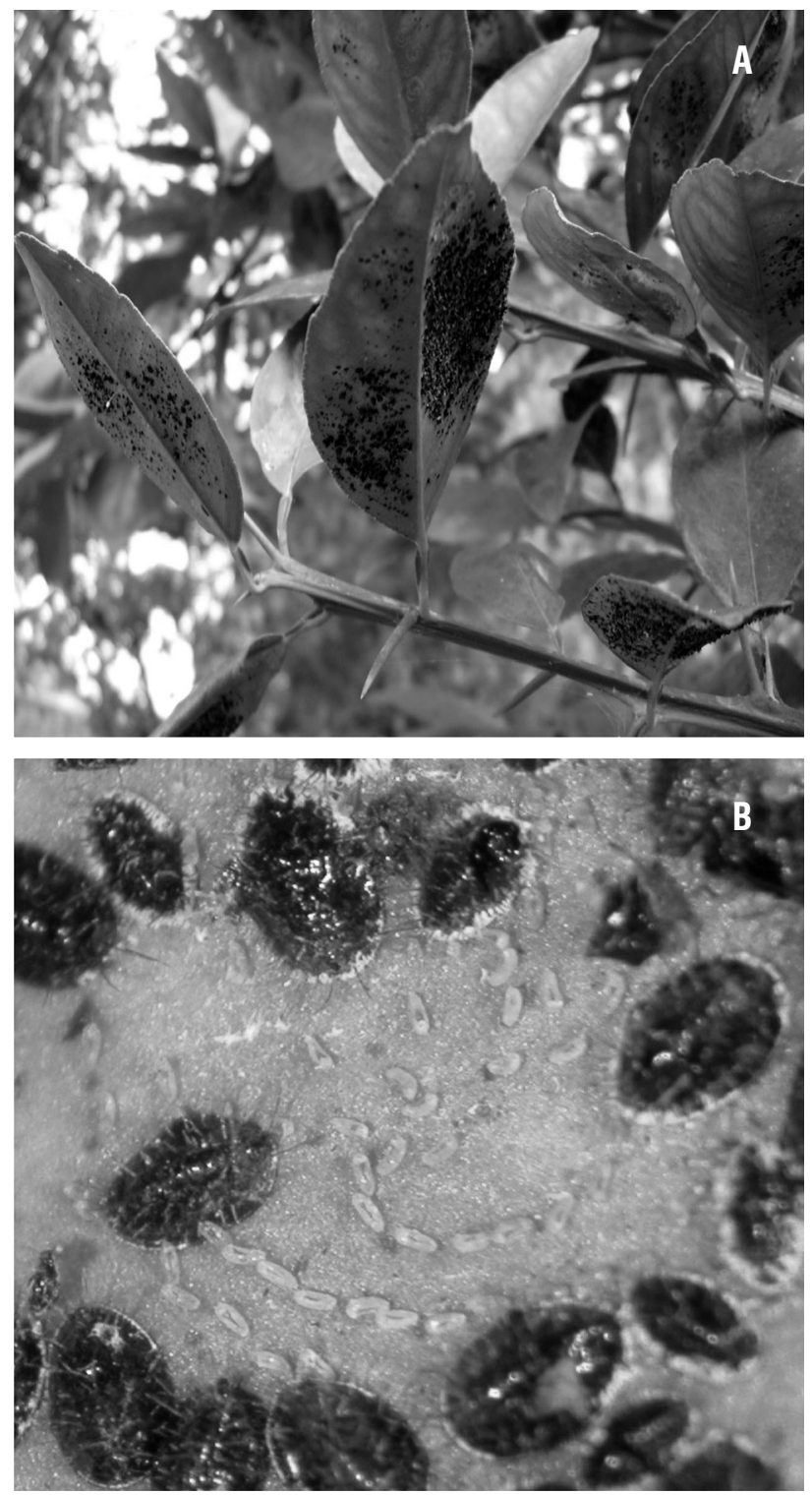

Figura 1 - Ninfas de mosca-negra Aleurocanthus woglumi (Hemiptera: Aleyrodidae) na área urbana de Manaus, Am. A) em folhas de citros; B) postura em espiral. 


\section{BIBLIOGRAFIA CITADA}

Angeles, N.; Oakley, R.; Osorio, J.O. 1968. Presencia em Venezuela de Aleurocanthus woglumi Ashby (Aleyrodidae: Homoptera) mosca prieta de los cítricos. Agronomia Tropical 18(4): $487-$ 488

Cunha, M.L.A. 2003. Distribuição geográfica, aspectos biológicos e controle quimico da mosca negra dos citros, Aleurocanthus woglumi Ashby (Hemiptera: Aleyrodidae), nas condiçôes ambientais do Estado do Pará. Dissertação de mestrado, Universidade Federal Rural da Amazônia, Belém/PA. 57pp.

Dietz, H.F.; Zetek, J. 1920. The blackfly of citrus and other subtropical plants. USDA Bulletin 885; 1-55.

Dowell, R.V., Cherry, R.H.; Fitzpatrick, G.E.; Reinert, J.A.; Knapp, J.L. 1981. Biology, plant-insect relations, and control of citrus blackfly. Florida Agricultural Experiment Station Bulletin, 818: $1-48$.

Jordão, A.L.; Silva, R.A. 2006. Guia de Pragas Agricolas para o Manejo Integrado no Estado do Amapá. Ribeirão Preto: Ed. Holos. 182pp.

Lemos, R. N. S.; Silva, G. S.; Araújo, J. R. G.; Chagas, E. F.; Moreira, A. A.;Soares, A. T. M. 2006. Ocorrência de Aleurocanthus woglumi Ashby (Hemiptera: Aleyrodidae) no Maranhão. Neotropical Entomology, (35): 4.

Maia, W.J.M.S.; Souza, J.C.; Marques, L.C.; Silva, L.M.S.; Benaduce, R.V.; Gentil, R.M. 2005. Infestação em citros por Aleurocanthus woglumi (Ashby) e perspectivas de controle biológico aplicado no Pará. Anais do $9^{\circ}$ Simpósio de Controle Biológico. Recife. p.183.
Newell, W.; A.C. Brown. 1939. Eradication of the citrus blackfly in Key West, Fla. Journal Economic Entomology, 32: 680-682.

Nguyen, R.; Hamon, A.B. 2003. Citrus Blackfly, Aleurocanthus woglumi Ashby (Homoptera: Aleyrodidae). University of Florida. CIR 360.

Oliveira, M.R.V.; Silva, C.C.A.; Návia, D.; De Paula, S.V. 2001. A mosca negra dos citros Aleurocanthus woglumi. Brasília, DF: Ministério da Agricultura, Pecuária e Abastecimento, (Alerta Quarentenário).

Pena, M.R.; Silva, N.M. 2007. Sugadora negra. In: Revista Cultivar: Hortaliças e Frutas. Pelotas/RS Ano VII, 41.16-18.

Pena, M.R., J.D. Vendramim, A.L. Lourenção, N.M. Silva, P.T. Yamamoto \& M.S. Gonçalves. 2008. Ocorrência da moscanegra-dos-citros, Aleurocanthus woglumi Ashby (Hemiptera: Aleyrodidae) no Estado de São Paulo. Revista de Agricultura. 83: 61-65.

Smith, H.D., Maltby, H.L.; Jimenez, E.J. 1964. Biological control of the citrus blackfly in Mexico. USDA-ARS, Technical Bulletin, 1311: 1-30.

Recebido em 11/04/2007

Aceito em 16/05/2008 
